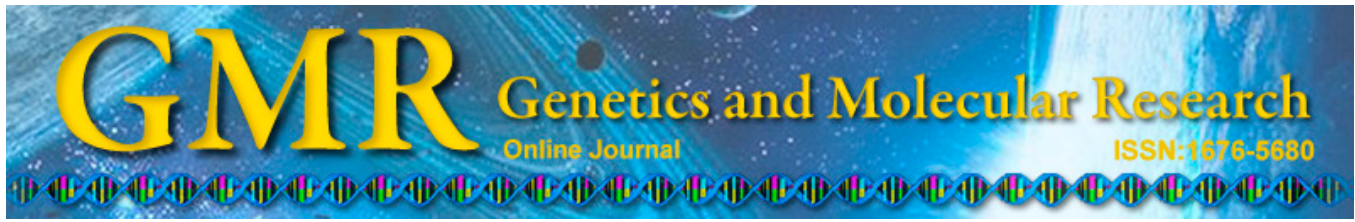

\title{
Effect of curcumin on p38MAPK expression in DSS-induced murine ulcerative colitis
}

\author{
C.-P. Li ${ }^{1}$, J.-H. Li' ${ }^{2}$, S.-Y. He', O. Chen ${ }^{3}$ and L. Shi ${ }^{1}$ \\ ${ }^{1}$ Department of Gastroenterology, \\ Affiliated Hospital of Luzhou Medical College, Luzhou, \\ Sichuan Province, China \\ ${ }^{2}$ West China School of Medicine, Sichuan University, Chengdu, \\ Sichuan Province, China \\ ${ }^{3}$ Department of Gastroenterology, People's Hospital of Ya'an City, \\ Sichuan Province, China \\ Corresponding author: C.-P. Li \\ E-mail: ChangPingLicn@163.com
}

Genet. Mol. Res. 14 (2): 3450-3458 (2015)

Received September 28, 2014

Accepted November 2, 2014

Published April 15, 2015

DOI http://dx.doi.org/10.4238/2015.April.15.8

ABSTRACT. The aim of this study was to determine the therapeutic
effect of curcumin on dextran sulfate sodium-induced ulcerative colitis
(UC) and to explore the related mechanism. Sixty mice were randomly
divided into 6 groups. A group was the normal control group; B group
was the model group; C group was the $1.5 \mathrm{mg} / \mathrm{kg}$ dexamethasone group
based on the B group; and D, E and F groups were 15,30 , and $60 \mathrm{mg} /$
kg curcumin groups, respectively, based on the B group. The mice were
killed 7 days after treatment; the expression of TNF- $\alpha$ and MPO in
colon tissue was determined with ELISA, and colon p-p38MAPK and
p38MAPK mRNA expression was evaluated by immunohistochemistry
and RT-PCR, respectively. In the C, D, E, and F groups, TNF- $\alpha$ and
MPO levels significantly decreased (P $<0.05)$, and the expression of
p-p38MAPK also significantly decreased (P $<0.01$ ). The expression of
p38MAPK mRNA in the C, D, E, and F groups decreased (P $<0.01$ ),
and there was a statistically significant difference between the E and F 
groups $(\mathrm{P}<0.01)$. Curcumin had a therapeutic effect, which probably played a role in UC treatment by inhibiting the p38MAPK signaling pathway, thereby reducing the release of TNF- $\alpha$.

Key words: Curcumin; Ulcerative colitis; p38 MAPK

\section{INTRODUCTION}

Ulcerative colitis (UC) is a chronic nonspecific inflammatory bowel disease, which is a kind of inflammatory bowel disease (IBD) with long duration; cure is difficult, and there is a tendency for canceration. Therefore, it is listed as one of the modern refractory diseases by WHO. Its etiology and pathogenesis have not yet been elucidated, where it may be connected with immunity, infection, genetics, and mental and environmental factors (Caprilli et al., 2007; Endo et al., 2009; Matricon, 2010). Currently, the commonly used traditional medicines in the treatment of UC have side effects such as ease of relapse after withdrawal and need for longterm medication (Martínez-Montiel and Muñoz-Yagüe, 2006). On the other hand, biological drug therapy often produces drug resistance in the treatment process, and costs are too high for it to be suitable for long-term and wide clinical use. Thus, the search for economic drugs with limited adverse reactions is of great significance. Curcumin is a natural polyphenol substance, extracted from the traditional Chinese medicine Rhizoma Curcumae Longae, and studies have confirmed that it has a variety of pharmacological effects and biological properties, such as antiinflammatory, antiinfective, antitumor, antioxidative, blood pressure-lowering and immune regulatory effects (Goel et al., 2008; Giri et al., 2009). This study aimed to determine the therapeutic effect of curcumin on dextran sulfate sodium (DSS)-induced murine UC, examining the effects of curcumin on the expression of TNF- $\alpha$, MPO, and p38 mitogen-activated protein kinase (p38MAPK) protein and mRNA, and to explore its therapeutic mechanism.

\section{MATERIAL AND METHODS}

\section{Material}

Sixty healthy BALB/c mice, all females, aged 6-7 weeks, weighing 16-20 g, were purchased from Chongqing TangXin Biological Technology Co. Ltd. DSS was purchased from Shanghai Sigma-Aidrich company (Shanghai), curcumin from Aladdin, and dexamethasone sodium phosphate injection from Affiliated Hospital of Luzhou Medical College. TNF- $\alpha$ and MPO ELISA kit were obtained from RD, American, phosphorylated p38MAPK mouse monoclonal antibody from Santa Cruz, and benzidine detection kit from Beijing Zhongshan Reagent Company. Trizol total RNA extraction and reverse transcription reagent kits were purchased from Takara, and the primer synthesis and probe modification were performed by Shanghai Biological Engineering Co., Ltd.

\section{Methods}

\section{Grouping and modeling}

Sixty healthy female BALB/c mice were adaptively fed for 1 week and then randomly 
divided into 6 groups, with 10 mice in each group as follows. A group: normal control group, with free access to drinking water, with intraperitoneal injection of $1 \mathrm{~mL}$ saline daily; B group: DSS model group, freely drinking 5\% DSS solution, with intraperitoneal injection of $1 \mathrm{~mL}$ $2.5 \%$ ethanol daily; $\mathrm{C}$ group: dexamethasone treatment group, with the same drinking solution as in B group, with intraperitoneal injection of dexamethasone once daily (at doses of 1.5 $\mathrm{mg} / \mathrm{kg}$ dissolved in $1 \mathrm{~mL}$ normal saline); $\mathrm{D}$ group: curcumin low dose group, with the same drinking solution as in B group, with intraperitoneal injection of curcumin solution once daily (at $15 \mathrm{mg} / \mathrm{kg}$ in $1 \mathrm{~mL} 2.5 \%$ ethanol solution); E Group: curcumin middle dose group, with the same drinking solution as in B group, with intraperitoneal injection of curcumin solution once daily (at $30 \mathrm{mg} / \mathrm{kg}$ in $1 \mathrm{~mL} \mathrm{2.5 \%}$ ethanol); F Group: curcumin high dose group, with the same drinking solution as in B group, with intraperitoneal injection of curcumin solution once daily (at $60 \mathrm{mg} / \mathrm{kg}$ in $1 \mathrm{~mL} 2.5 \%$ ethanol). The whole drug therapy period was 7 days. All mice were then euthanized, and the colon collected, part for embedding in paraffin and part for storage in liquid nitrogen.

\section{Evaluation of colonic inflammation}

The disease activity index (DAI) and histological injury scores were used to evaluate the colitis. 1) DAI $=($ weight loss percentage score + stool score + hematochezia score $) / 3$ (Swee et al., 2006; Mao et al., 2012). The weight loss percentage score was divided into 5 grades: no decrease: 0 point; decrease 1-5\%: 1 point; decrease $6-10 \%$ : 2 points; decrease 11 15\%: 3 points; decrease $>15 \%$ : 4 points; stool: normal: 0 points; semi-loose: 2 points; loose: 4 points; fecal occult blood: negative: 0 point; positive: 2 points; bloody stools: 4 points. 2) Histopathological score (Murano et al., 2000; Tjandra et al., 2002; Osman et al., 2004), based on HE staining and examination under a light microscope. Each intestinal tissue specimen was evaluated using 3 visual fields, and the mean value of these 3 fields was rated: mucosa was normal, without damage: 0 point; crypt glands 1/3 lost: 1 point; crypt glands $2 / 3$ lost: 2 points; crypt glands all lost, presence of lamina propria covering simple epithelium, accompanied by inflammatory cell infiltration: 3 points; mucosal membrane dissipated and destroyed, accompanied by significant inflammatory cell infiltration: 4 points.

\section{Colonic TNF- $\alpha$ and MPO levels detection by ELISA assay}

We took 1-cm colon tissue, added a certain amount of phosphate buffer then made tissue homogenate, centrifuged and the supernatant was performed according to ELISA kit instructions.

\section{Colon p-p38MAPK protein expression by immunohistochemical assay}

In accordance with the SP immunohistochemical staining kit instructions, the positive criteria were as follows: the nucleus and/or cytoplasm appeared tan brown or brownish yellow. The semi-quantitative method was used for grading, according to Maltby et al. (2010), where staining intensity was divided into 4 levels: no staining: 0 point; yellowish, 1 point; brownish yellow: 2 points, brown: 3 points. Each slice was graded at 5 levels according to the percentage of positive cells: none: 0 ; positive staining cells accounted for 1-10\%: 1 point; positive cells accounted for $11-50 \%$ : 2 points; positive cells accounted for $51-75 \%$ : 3 points; positive cells accounted for $76-100 \%$ : 4 points. The score of each section was the product of the above scores. 


\section{Colon p38MAPK mRNA expression detection by RT-PCR}

Total RNA of mouse colon tissue was extracted with Trizol reagent according to instructions, and the purity and concentration of RNA were assessed with an ultraviolet spectrophotometer. Reverse transcription was performed to synthesize cDNA, which was then amplified by PCR. The amplified products were subjected to $1.0 \%$ agarose gel electrophoresis. The gray value of each band was analyzed by gel imaging, and the relative content of mRNA was expressed as semi-quantitative RT-PCR results by the ratio of sample target p38MAPK gene to the internal reference. The primers used were GAPDH gene upstream primer sequence: 5'-TGC TGT CCC TGT ATG CCT CTG-3' and downstream primer sequence: 5'-TTG ATG TCA CGC ACG ATT TCC-3' (223 bp); and p38MAPK gene upstream primer sequence: 5'-GAG ACC GTT TCA GTC CAT C-3' and downstream primer sequence: 5'-ATT CCT CCA GTG ACC TTG C-3' (128 bp). p38MAPK amplification conditions were: $94^{\circ} \mathrm{C}$ for $5 \mathrm{~min}, 30$ cycles of $94^{\circ} \mathrm{C}$ for $35 \mathrm{~s}, 54^{\circ} \mathrm{C}$ for $30 \mathrm{~s}$ and $72^{\circ} \mathrm{C}$ for $30 \mathrm{~s}$, and a final extension at $72^{\circ} \mathrm{C}$ for $5 \mathrm{~min}$.

\section{Statistical analysis}

The SPSS 11.5 statistical software was used for the data processing, and all data are reported as means $\pm \mathrm{SD}$. After the data were tested for homogeneity of variance, one-way ANOVA was performed. The LSD-t test was used for intergroup comparison, and linear correlation analysis was carried out. $\mathrm{P}<0.05$ was considered to be statistically significant.

\section{RESULTS}

\section{DAI score}

The basic activities of the A group were normal, without weight loss or hematochezia, while the mice in other groups gradually showed a slow response, i.e., easily frightened, anorexia, weight loss, reduced water consumption, stool attenuate and bloody purulent stool, etc., after the intake of $5 \%$ DSS. However, the symptoms of the C, D, E, and F groups were significantly milder than in the B group, and DAI scores were also significantly lower than in the B group, with statistically significant difference $(\mathrm{P}<0.01)$. There was a statistically significant difference between the $\mathrm{C}$ group and $\mathrm{D}$ and $\mathrm{E}$ groups $(\mathrm{P}<0.05)$, and also, there was a significant difference between the $\mathrm{D}$ group and $\mathrm{E}$ and $\mathrm{F}$ groups $(\mathrm{P}<0.05)$, while there was no significant difference between the $\mathrm{C}$ group and $\mathrm{F}$ and $\mathrm{E}$ groups $(\mathrm{P}>0.05)$ (Table 1).

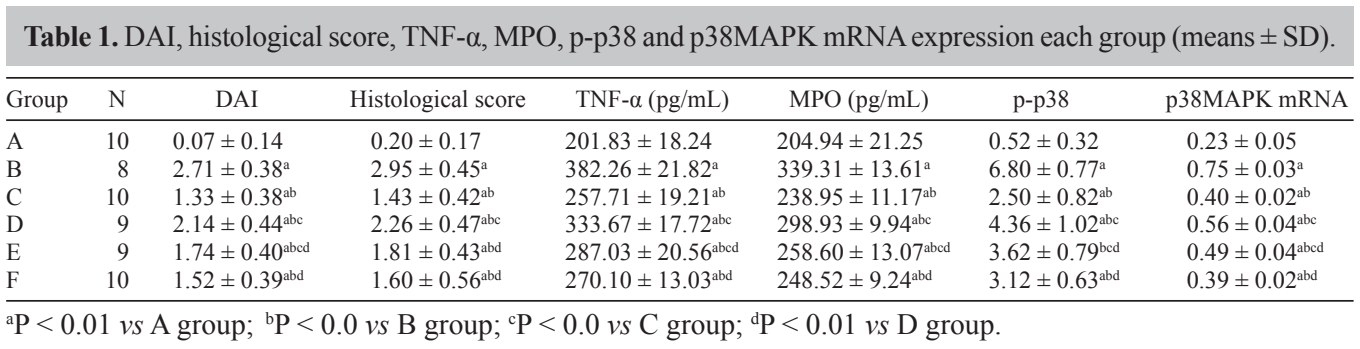




\section{Histological score}

The histological scores determined under a light microscope are given in Table 1 and Figure 1. The histological scores of the C, D, E, and F groups were lower than for the B group, with a significant difference $(\mathrm{P}<0.01)$. The difference was statistically significant between the $\mathrm{C}$ and $\mathrm{D}$ groups $(\mathrm{P}<0.01)$, as well as between the $\mathrm{D}$ group and $\mathrm{E}$ and $\mathrm{F}$ groups $(\mathrm{P}<0.05)$.
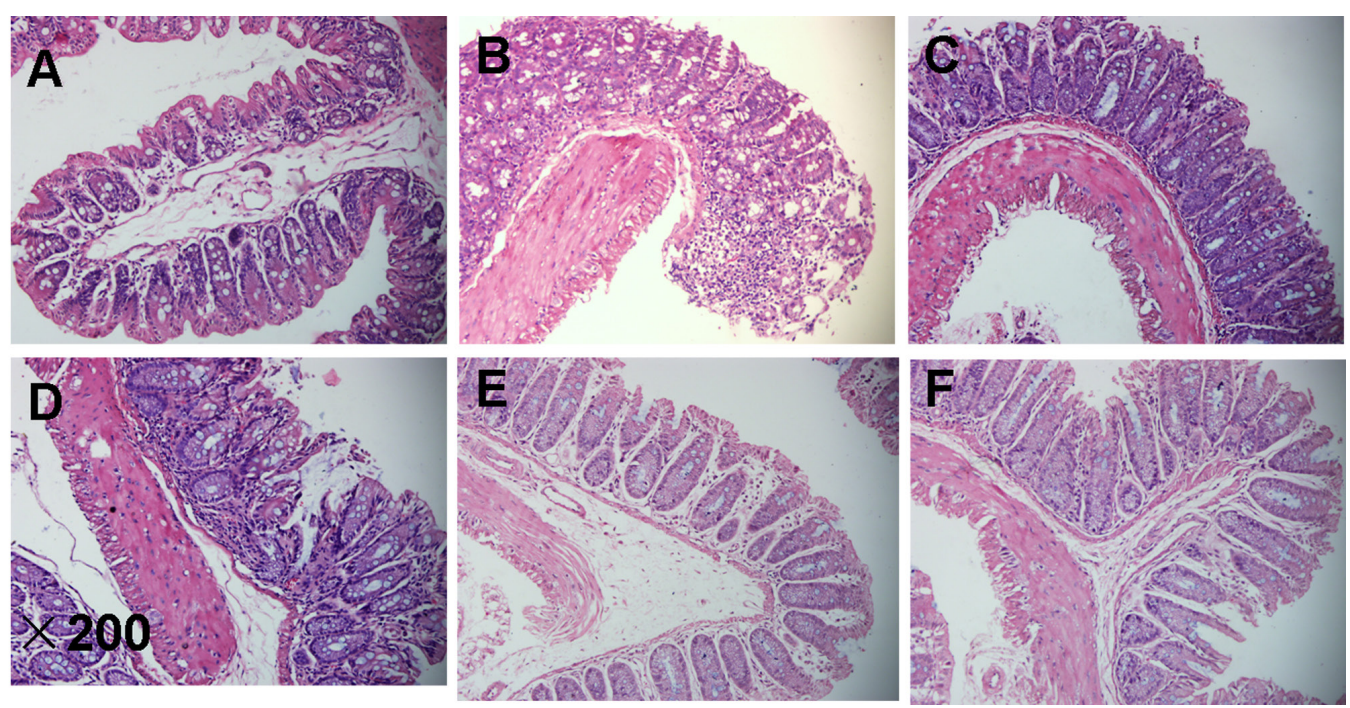

Figure 1. Colon tissue observation under the microscopy (HE x 200).

\section{TNF- $\alpha$, MPO levels of colonic mucosa}

In the B group, TNF- $\alpha$ and MPO levels of colon tissue were significantly higher than in the A group, while TNF- $\alpha$ and MPO levels of the C, D, E, and F groups were lower than for the B group, with a statistically significant difference $(\mathrm{P}<0.01)$. There was a statistically significant difference between the $\mathrm{C}$ group and $\mathrm{D}$ and $\mathrm{E}$ groups $(\mathrm{P}<0.05)$, as well as between the $\mathrm{D}$ group and $\mathrm{E}$ and $\mathrm{F}$ groups $(\mathrm{P}<0.05)$ (Table 1).

\section{Colonic tissue p-p38MAPK expression}

p38MAPK expression was mainly in the epithelial cells of the mucous layer and mononuclear cells and neutrophils infiltrating the submucous (Figure 2). In the A group, there was less expression in the cytoplasm and the nucleus, and the positive cells were few. In the B group, the cytoplasm and nucleus were strongly stained, mainly in the nucleus, and the color was brown. In the $\mathrm{C}, \mathrm{D}, \mathrm{E}$, and $\mathrm{F}$ groups, the expression of p-p38MAPK-positive cells was reduced and the staining was lighter, mainly nuclear and scattered cytoplasmic expression; the score showed significant differences when compared with the B group $(\mathrm{P}<0.01)$. The score of the $\mathrm{C}$ group was statistically different from that of $\mathrm{D}$ and $\mathrm{E}$ groups $(\mathrm{P}<0.01)$, and there was a statistically significant difference between the $\mathrm{D}$ group and $\mathrm{E}$ and $\mathrm{F}$ groups $(\mathrm{P}<0.05$, Table 1$)$. 

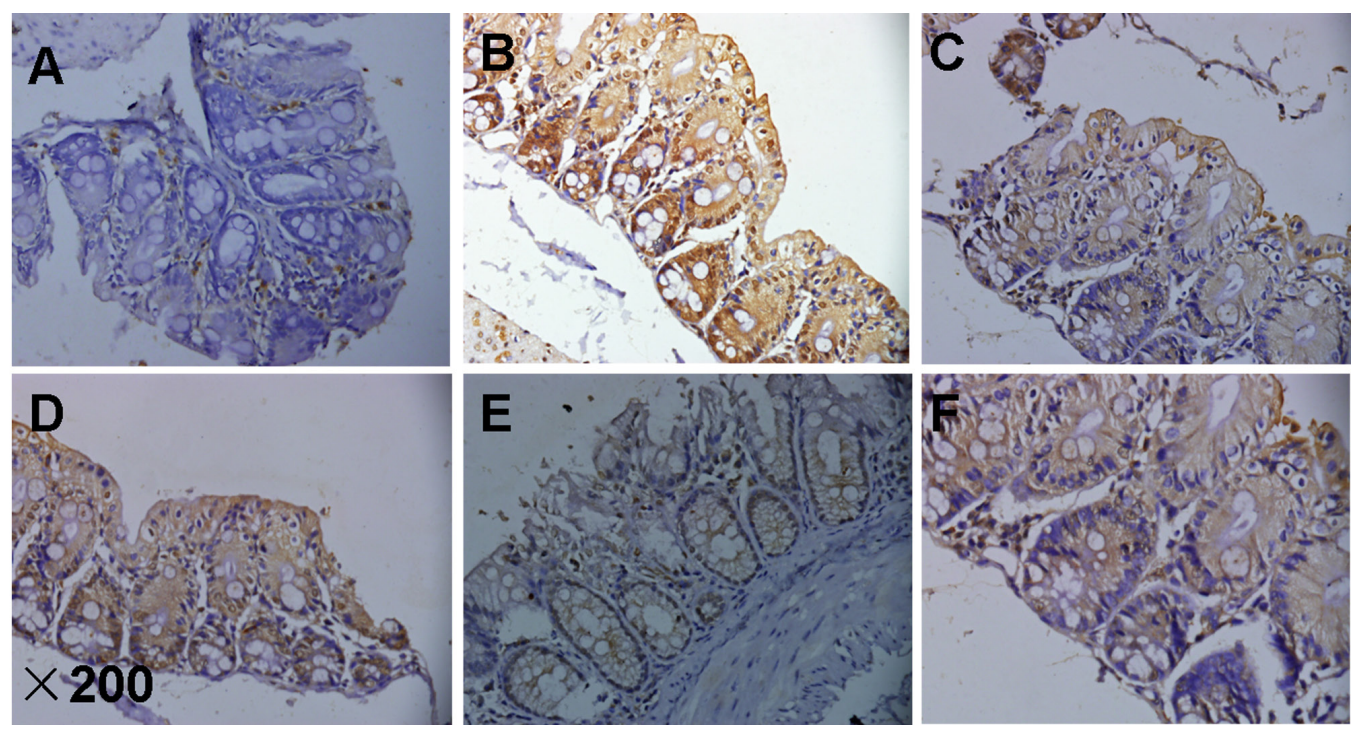

Figure 2. p-p38MAPK immunohistochemical expression situation of colon tissue (SP x 400).

\section{Colon tissue p38MAPK mRNA expression}

RT-PCR (Figure 3) exhibited the following results. Compared with the A group, the colon mucosa $\mathrm{p} 38 \mathrm{MAPK}$ mRNA expression level of the B group significantly increased (P $<0.01$ ), while p38MAPK mRNA expression levels in mucosal tissues of the C, D, E, and F groups decreased compared with the $\mathrm{B}$ group, with a statistically significant difference $(\mathrm{P}<$ 0.01 ). There was a statistically significant difference between the $\mathrm{C}$ group and the $\mathrm{D}$ and $\mathrm{E}$ groups $(\mathrm{P}<0.01)$, and the colonic tissue expression of the $\mathrm{D}$ group was significantly higher than that of the $\mathrm{E}$ and $\mathrm{F}$ groups, with a significant difference $(\mathrm{P}<0.01$, Table 1$)$.

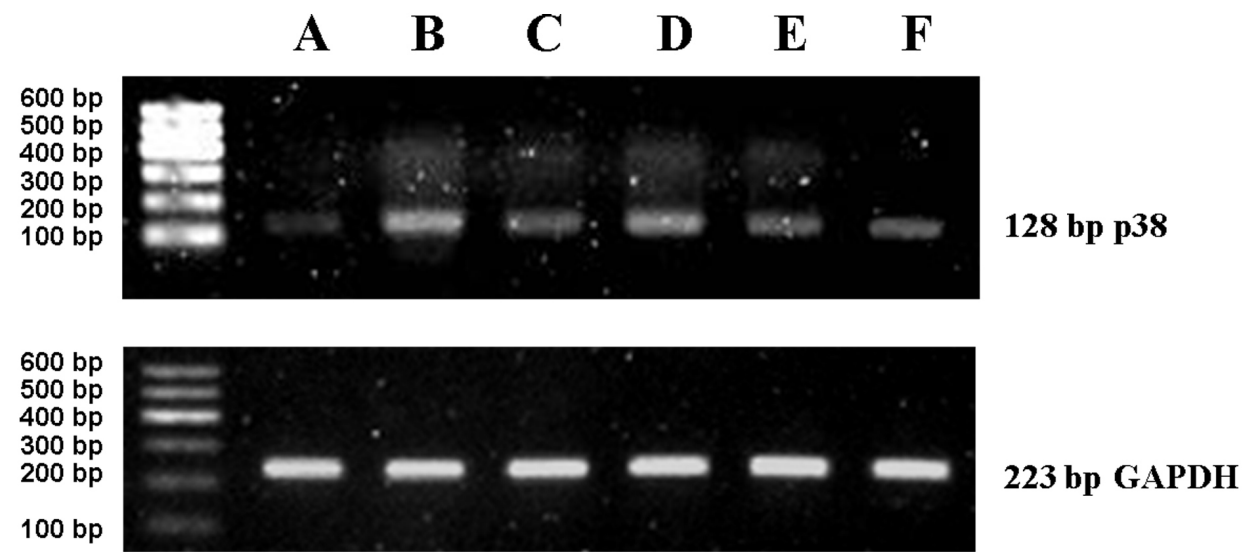

Figure 3. p38MAPK mRNA expression of colon tissue in each group: Lane A: A group; lane B: B group; lane C: C group; lane D: D group; lane E: E group; lane F: F group. 


\section{DISCUSSION}

UC is a chronic non-specific colitis, mainly appearing as inflammatory bowel disease and ulcers. Its cause is still unknown, but the effects of cytokines in the pathogenesis, development and prognosis have been recognized. The disrupted balance between proinflammatory cytokines and antiinflammatory cytokines plays a key role (Eaden et al., 2000; Li et al., 2009), where TNF- $\alpha$, as an important proinflammatory cytokine, and immunomodulatory factors are mainly generated by the autocrine and paracrine regulation of monocytes and macrophages. It could promote the release of other inflammatory cytokines, and amplify the inflammatory reaction, increase the accumulation of other chemical factors to neutrophils, increase the expression of endothelial cell adhesion molecule, induce specific enzyme-mediated tissue injury, increase intestinal permeability and ion transport (which would cause diarrhea), and thus participate in UC inflammation and immune response. TNF- $\alpha$ is recognized as an important inflammatory cytokine closely associated with UC pathogenesis (Papadakis and Targan, 2000), and studies have also confirmed that TNF- $\alpha$ expression would significantly increase in the UC active phase, playing an important role in the pathogenesis and development of UC. Myeloperoxidase (MPO) is an important peroxidase produced by neutrophils, mainly existing in neutrophils and monocytes. It could be defined as a specific marker of neutrophils, and so any tissue with the presence of neutrophil infiltration could be evaluated for infiltration number and activity of inflammatory cells by measuring MPO activity. Therefore, MPO activity in intestinal mucosa could be used as a monitoring indicator of disease severity in UC patients (Park et al., 2012).

p38MAPK is an important member of the MAPK family (Zarubin and Han, 2005), widely present in various tissues, where under physiological conditions, p38 is mainly located in the cytoplasm, showing very low activity. It could be activated by various cytokines (TNF- $\alpha$, IL-1), lipopolysaccharide, stress stimuli (heat shock, hyperosmolar injury, ischemia - reperfusion) (Rafiee et al., 2002; Jameel et al., 2009). A number of studies have shown that cells exposed to the above stimuli could activate p38MAPK, which would then regulate the inflammatory response by the following means. Inflammatory stimuli could activate p38MAPK, and p38MAPK could also affect the production of TNF- $\alpha$, IL-1, IL-6, IL-8 and other proinflammatory cytokines, and the inflammation-inhibitory cytokine IL-10, affecting the balance of proinflammatory and antiinflammatory cytokines, thus influencing the inflammatory process. p38 could mediate the activation of neutrophils; the proinflammatory cytokines (TNF- $\alpha$, etc) could stimulate neutrophils and then activate p38MAPK; and the cytokines could significantly enhance neutrophil respiratory burst effect, thereby amplifying and sustaining the inflammatory response, leading to continuous inflammation response and injury. p38 could induce the expression of intracellular nitric oxide (NO), increase the intracellular levels of inducible nitric oxide synthase (iNOS), involved in inflammation and tissue damage. p38 also plays an important role in the repair of cells and tissues (Kanaji et al., 2012), thus affecting the antiinflammatory capabilities of tissues. p38 could positively regulate NF- $\mathrm{KB}$ activity, by

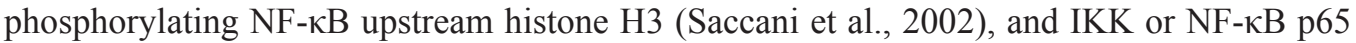
activity, thereby controlling the release of inflammatory cytokines. Therefore, the p38 MAPK signal transduction pathway plays an important role in the inflammatory response. Rafiee et al. (2002) found that p-p38MAPK was higher in IBD patients than in normal people, and positively correlated with the degree of intestinal inflammation (Zhao et al., 2011). Waetzig et al. (2002) found that during IBD intestinal epithelial injury, the activity of p38MAPK was 
significantly increased, and revealed that by tissue immunohistochemistry high p38MAPK expression in macrophages and neutrophils of the intestinal lamina propria. When SB20358O, a p38MAPK inhibitor, was added to cultured mucosal tissue of IBD patients, the TNF- $\alpha$ secretion of mucosal tissue significantly decreased. Docena et al. (2010) also found that the mucosal p-p38MAPK level of IBD patients increased, after exposure of cultivated IBD mucosa lesions to 4 different p38MAPK inhibitors, and that the p-p38MAPK levels declined, while the expressions of TNF- $\alpha$, IL- $1 \beta$ and IL- 6 also reduced. The above studies illustrated that p38MAPK signal transduction plays an important role in the pathogenesis of IBD.

Curcumin is a natural polyphenol extracted from Rhizoma Curcumae Longae. Because of its pharmacological effects such as antiinflammatory, antiinfective, antioxidant, immunomodulatory and antitumor effects, it is widely used to treat cardiovascular and cerebrovascular diseases, lung damage, cancer and other diseases. This study found that curcumin improved DAI and histological injury in DSS-induced mice acute phase, while TNF- $\alpha$ and MPO levels of colonic mucosa in the curcumin treatment group decreased, and expression of p-p38MAPK and p38MAPK mRNA was also significantly reduced, confirming that curcumin had some therapeutic effect against murine UC. The reasons could be: 1) inhibition of the release of the proinflammatory cytokine TNF- $\alpha$; 2 decrease in neutrophil infiltration, thereby reducing damage from intestinal mucosal inflammation; and 3) inhibition of the expression of p38MAPK and its upstream and downstream signaling pathways. Curcumin could partially inhibit the activating signals of p38MAPK, such as cytokines (TNF- $\alpha$, IL-6), and the stimulation of irritability to play a role in treatment of UC. The study also found that the effects with a high dose of curcumin were better than with low and middle doses, and when compared with the dexamethasone group, there was no significant difference in the treatment of UC, indicating that curcumin worked in a dose-dependent manner, with the high dose being most effective.

In conclusion, curcumin has a good therapeutic effect towards UC, probably by inhibiting the p38MAPK signaling pathway, reducing the release of TNF- $\alpha$ and other proinflammatory cytokines, and reducing neutrophil infiltration, thereby reducing inflammation injury in the mouse intestine and playing a role in UC therapy. Notably, UC has a long duration, with recurrence and a certain risk of canceration. Curcumin has an antitumor effect, and could therefore prevent canceration. Moreover, curcumin is safe and inexpensive, and could lead to the development of a new generation of therapeutic agents for UC.

\section{ACKNOWLEDGMENTS}

Research supported by the Science and Technology Department of Sichuan Province \#2011SZ0094.

\section{REFERENCES}

Caprilli R, Viscido A and Latella G (2007). Current management of severe ulcerative colitis. Nat. Clin. Pract. Gastroenterol. Hepatol. 4: 92-101.

Docena G, Rovedatti L, Kruidenier L, Fanning A, et al. (2010). Down-regulation of p38 mitogen-activated protein kinase activation and proinflammatory cytokine production by mitogen-activated protein kinase inhibitors in inflammatory bowel disease. Clin. Exp. Immunol. 162: 108-115.

Eaden J, Abrams K, Ekbom A, Jackson E, et al. (2000). Colorectal cancer prevention in ulcerative colitis: a case-control study. Aliment. Pharmacol. Ther. 14: 1451-1531.

Endo K, Shiga H, Kinouchi Y and Shimosegawa T (2009). Inflammatory bowel disease: IBD. Rinsho Byori. 57: 527-532. 
Giri B, Gomes A, Sengupta R, Banerjee S, et al. (2009). Curcumin synergizes the growth inhibitory properties of Indian toad (Bufo melanostictus Schneider) skin-derived factor (BM-ANF1) in HCT-116 colon cancer cells. Anticancer Res. 29: 395-401.

Goel A, Kunnumakkara AB and Aggarwal BB (2008). Curcumin as "Curecumin": from kitchen to clinic. Biochem. Pharmacol. 75: 787-809.

Jameel NM, Thirunavukkarasu C, Wu T, Watkins SC, et al. (2009). p38-MAPK- and caspase-3-mediated superoxideinduced apoptosis of rat hepatic stellate cells: reversal by retinoic acid. J. Cell Physiol. 218: 157-166.

Kanaji N, Nelson A, Allen-Gipson DS, Sato T, et al. (2012). The p38 mitogen-activated protein kinases modulate endothelial cell survival and tissue repair. Inflamm. Res. 61: 233-244.

Li CS, Zhang Q, Lee KJ, Cho SW, et al. (2009). Interleukin-27 polymorphisms are associated with inflammatory bowel diseases in a Korean population. J. Gastroenterol. Hepatol. 24: 1692-1696.

Maltby S, Wohlfarth C, Gold M, Zbytnuik L, et al. (2010). CD34 is required for infiltration of eosinophils into the colon and pathology associated with DSS-induced ulcerative colitis. Am. J. Pathol. 177: 1244-1254.

Mao JW, Tang HY, Tan XY and Wang YD (2012). Effect of Etiasa on the expression of matrix metalloproteinase-2 and tumor necrosis factor- $\alpha$ in a rat model of ulcerative colitis. Mol. Med. Report. 6: 996-1000.

Martínez-Montiel MP and Muñoz-Yagüe MT (2006). Biologic therapies for chronic inflammatory bowel disease. Rev. Esp. Enferm. Dig. 98: 265-291.

Matricon J (2010). Immunopathogenesis of inflammatory bowel disease. Med. Sci. 26: 405-410.

Murano M, Maemura K, Hirata I, Toshina K, et al. (2000). Therapeutic effect of intracolonically administered nuclear factor kappa B (p65) antisense oligonucleotide on mouse dextran sulphate sodium (DSS)-induced colitis. Clin. Exp. Immunol. 120: 51-58.

Osman N, Adawi D, Ahrne S, Jeppsson B, et al. (2004). Modulation of the effect of dextran sulfate sodium-induced acute colitis by the administration of different probiotic strains of Lactobacillus and Bifidobacterium. Dig. Dis. Sci. 49: 320-327.

Papadakis KA and Targan SR (2000). Role of cytokines in the pathogenesis of inflammatory bowel disease. Annu. Rev. Med. 51: 289-296.

Park MY, Ji GE and Sung MK (2012). Dietary kaempferol suppresses inflammation of dextran sulfate sodium-induced colitis in mice. Dig. Dis. Sci. 570: 355-363.

Rafiee P, Johnson CP, Li MS, Ogawa H, et al. (2002). Cyclosporine A enhances leukocyte binding by human intestinal microvascular endothelial cells through inhibition of p38 MAPK and iNOS. Paradoxical proinflammatory effect on the microvascular endothelium. J. Biol. Chem. 277: 35605-35615.

Saccani S, Pantano S and Natoli G (2002). p38-Dependent marking of inflammatory genes for increased NF-kappa B recruitment. Nat. Immunol. 3: 69-75.

Swee M, McGuire JK, Wang Y, et al. (2006). Matrilysin controls neutrophil location in ulcerative colitis. Matrix Biol. 25: S48.

Tjandra K, Le T and Swain MG (2002). Experimental colitis attenuates development of toxin-induced cholangitis in rats. Dig. Dis. Sci. 47: 1216-1223.

Waetzig GH, Seegert D, Rosenstiel P, Nikolaus S, et al. (2002). p38 mitogen-activated protein kinase is activated and linked to TNF- $\alpha$ signaling in inflammatory bowel disease. J. Immunol. 168: 5342-5351.

Zarubin T and Han JH (2005). Activation and signaling of the p38 MAP kinase pathway. Cell Res. 15: 11-18.

Zhao XM, Kang B, Lu CL, Liu SQ, et al. (2011). Evaluation of p38 MAPK pathway as a molecular signature in ulcerative colitis. J. Proteome Res. 10: 2216-2225. 